\title{
Complete Measurement of the $\Lambda$ Electromagnetic Form Factors
}

M. Ablikim, ${ }^{1}$ M. N. Achasov ${ }^{10, d}$ P. Adlarson ${ }^{59}$ S. Ahmed,${ }^{15}$ M. Albrecht, ${ }^{4}$ M. Alekseev ${ }^{58 a, 58 c}$ A. Amoroso, ${ }^{58 a, 58 c}$ F. F. An ${ }^{1}$ Q. An ${ }^{55,43}$ Y. Bai, ${ }^{42}$ O. Bakina, ${ }^{27}$ R. Baldini Ferroli, ${ }^{23 a}$ Y. Ban,${ }^{35}$ K. Begzsuren, ${ }^{25}$ J. V. Bennett,${ }^{5}$ N. Berger, ${ }^{26}$ M. Bertani, ${ }^{23 a}$

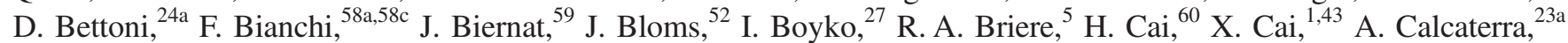
G. F. Cao ${ }^{1,47}$ N. Cao, ${ }^{1,47}$ S. A. Cetin,${ }^{46 b}$ J. Chai ${ }^{58 c}$ J. F. Chang,,${ }^{1,43}$ W. L. Chang, ${ }^{1,47}$ G. Chelkov, ${ }^{27, b, c}$ D. Y. Chen, ${ }^{6}$ G. Chen, ${ }^{1}$ H. S. Chen, ${ }^{1,47}$ J. C. Chen, ${ }^{1}$ M. L. Chen, ${ }^{1,43}$ S. J. Chen, ${ }^{33}$ Y. B. Chen, ${ }^{1,43}$ W. Cheng, ${ }^{58 \mathrm{c}}$ G. Cibinetto, ${ }^{24 \mathrm{a}}$ F. Cossio, ${ }^{58 \mathrm{c}}$ X. F. Cui ${ }^{34}$ H. L. Dai, ${ }^{1,43}$ J. P. Dai,${ }^{38, h}$ X. C. Dai, ${ }^{1,47}$ A. Dbeyssi, ${ }^{15}$ D. Dedovich, ${ }^{27}$ Z. Y. Deng, ${ }^{1}$ A. Denig, ${ }^{26}$ I. Denysenko, ${ }^{27}$ M. Destefanis, ${ }^{58 a, 58 c}$ F. De Mori, ${ }^{58 a, 58 c}$ Y. Ding, ${ }^{31}$ C. Dong, ${ }^{34}$ J. Dong, ${ }^{1,43}$ L. Y. Dong, ${ }^{1,47}$ M. Y. Dong, ${ }^{1,43,47}$ Z. L. Dou, ${ }^{33}$ S. X. Du, ${ }^{63}$ J. Z. Fan, ${ }^{45}$ J. Fang, ${ }^{1,43}$ S. S. Fang, ${ }^{1,47}$ Y. Fang, ${ }^{1}$ R. Farinelli, ${ }^{24 a, 24 b}$ L. Fava, ${ }^{58 b, 58 c}$ F. Feldbauer, ${ }^{4}$ G. Felici, ${ }^{23 a}$ C. Q. Feng, ${ }^{55,43}$ M. Fritsch, ${ }^{4}$ C. D. Fu, ${ }^{1}$ Y. Fu, ${ }^{1}$ Q. Gao, ${ }^{1}$ X. L. Gao, ${ }^{55,43}$ Y. Gao,${ }^{56}$ Y. Gao, ${ }^{45}$ Y. G. Gao, ${ }^{6}$ Z. Gao, ${ }^{55,43}$ B. Garillon, ${ }^{26}$ I. Garzia, ${ }^{24 a}$ E. M. Gersabeck, ${ }^{50}$ A. Gilman, ${ }^{51}$ K. Goetzen, ${ }^{11}$ L. Gong, ${ }^{34}$ W. X. Gong, ${ }^{1,43}$ W. Gradl, ${ }^{26}$ M. Greco, ${ }^{58 a, 58 c}$ L. M. Gu, ${ }^{33}$ M. H. Gu, ${ }^{1,43}$ S. Gu, ${ }^{2}$ Y. T. Gu, ${ }^{13}$ A. Q. Guo, ${ }^{22}$ L. B. Guo, ${ }^{32}$ R. P. Guo, ${ }^{36}$ Y. P. Guo, ${ }^{26}$ A. Guskov ${ }^{27}$ S. Han,${ }^{60}$ X. Q. Hao, ${ }^{16}$ F. A. Harris ${ }^{48}$ K. L. He ${ }^{1,47}$ F. H. Heinsius, ${ }^{4}$ T. Held,${ }^{4}$ Y. K. Heng, ${ }^{1,43,47}$ Y. R. Hou ${ }^{47}$ Z. L. Hou, ${ }^{1}$ H. M. Hu, ${ }^{1,47}$ J. F. Hu, ${ }^{38, h}$ T. Hu, ${ }^{1,43,47}$ Y. Hu, ${ }^{1}$ G. S. Huang, ${ }^{55,43}$ J. S. Huang, ${ }^{16}$ X. T. Huang, ${ }^{37}$ X. Z. Huang ${ }^{33}$ N. Huesken, ${ }^{52}$ T. Hussain,${ }^{57}$ W. Ikegami Andersson, ${ }^{59}$ W. Imoehl, ${ }^{22}$ M. Irshad,${ }^{55,43}$ Q. Ji, ${ }^{1}$ Q. P. Ji, ${ }^{16}$ X. B. Ji, ${ }^{1,47}$ X. L. Ji, ${ }^{1,43}$ H. L. Jiang, ${ }^{37}$ X. S. Jiang, ${ }^{1,43,47}$ X. Y. Jiang, ${ }^{34}$ J. B. Jiao, ${ }^{37}$ Z. Jiao, ${ }^{18}$ D. P. Jin, ${ }^{1,43,47}$ S. Jin, ${ }^{33}$ Y. Jin, ${ }^{49}$ T. Johansson, ${ }^{59}$

N. Kalantar-Nayestanaki, ${ }^{29}$ X. S. Kang, ${ }^{31}$ R. Kappert, ${ }^{29}$ M. Kavatsyuk, ${ }^{29}$ B. C. Ke, ${ }^{1}$ I. K. Keshk, ${ }^{4}$ T. Khan, ${ }^{55,43}$

A. Khoukaz, ${ }^{52}$ P. Kiese, ${ }^{26}$ R. Kiuchi, ${ }^{1}$ R. Kliemt,${ }^{11}$ L. Koch, ${ }^{28}$ O. B. Kolcu, ${ }^{46 b, f}$ B. Kopf, ${ }^{4}$ M. Kuemmel, ${ }^{4}$ M. Kuessner, ${ }^{4}$ A. Kupsc, ${ }^{59}$ M. Kurth, ${ }^{1}$ M. G. Kurth, ${ }^{1,47}$ W. Kühn, ${ }^{28}$ J. S. Lange, ${ }^{28}$ P. Larin, ${ }^{15}$ L. Lavezzi,${ }^{58 c}$ H. Leithoff, ${ }^{26}$ T. Lenz,${ }^{26}$ C. Li,${ }^{59}$ Cheng Li ${ }^{55,43}$ D. M. Li ${ }^{63}$ F. Li, ${ }^{1,43}$ F. Y. Li, ${ }^{35}$ G. Li, ${ }^{1}$ H. B. Li,${ }^{1,47}$ H. J. Li, ${ }^{9, j}$ J. C. Li,${ }^{1}$ J. W. Li, ${ }^{41} \mathrm{Ke} \mathrm{Li},{ }^{1} \mathrm{~L} . \mathrm{K} . \mathrm{Li},{ }^{1} \mathrm{Lei} \mathrm{Li},{ }^{3}$ P. L. Li, ${ }^{55,43}$ P. R. Li, ${ }^{30}$ Q. Y. Li, ${ }^{37}$ W. D. Li, ${ }^{1,47}$ W. G. Li, ${ }^{1}$ X. H. Li, ${ }^{55,43}$ X. L. Li, ${ }^{37}$ X. N. Li ${ }^{1,43}$ X. Q. Li, ${ }^{34}$ Z. B. Li, ${ }^{44}$ Z. Y. Li, ${ }^{44}$ H. Liang, ${ }^{1,47}$ H. Liang, ${ }^{55,43}$ Y. F. Liang, ${ }^{40}$ Y. T. Liang, ${ }^{28}$ G. R. Liao, ${ }^{12}$ L. Z. Liao, ${ }^{1,47}$ J. Libby, ${ }^{21}$ C. X. Lin, ${ }^{44}$ D. X. Lin ${ }^{15}$ Y. J. Lin, ${ }^{13}$ B. Liu ${ }^{38, h}$ B. J. Liu, ${ }^{1}$ C. X. Liu, ${ }^{1}$ D. Liu,${ }^{55,43}$ D. Y. Liu,${ }^{38, h}$ F. H. Liu, ${ }^{39}$ Fang Liu, ${ }^{1}$ Feng Liu ${ }^{6}$ H. B. Liu, ${ }^{13}$ H. M. Liu,${ }^{1,47}$ Huanhuan Liu, ${ }^{1}$ Huihui Liu, ${ }^{17}$ J. B. Liu,,${ }^{55,43}$ J. Y. Liu, ${ }^{1,47}$ K. Y. Liu, ${ }^{31}$ Ke Liu, ${ }^{6}$ Q. Liu, ${ }^{47}$ S. B. Liu ${ }^{55,43}$ T. Liu,${ }^{1,47}$ X. Liu, ${ }^{30}$ X. Y. Liu, ${ }^{1,47}$ Y. B. Liu, ${ }^{34}$ Z. A. Liu, ${ }^{1,43,47}$ Zhiqing Liu, ${ }^{37}$ Y. F. Long, ${ }^{35}$ X. C. Lou,,${ }^{1,43,47}$ H. J. Lu, ${ }^{18}$ J. D. Lu, ${ }^{1,47}$ J. G. Lu, ${ }^{1,43}$ Y. Lu, ${ }^{1}$ Y. P. Lu, ${ }^{1,43}$ C. L. Luo, ${ }^{32}$ M. X. Luo, ${ }^{62}$ P. W. Luo, ${ }^{44}$ T. Luo, ${ }^{9, j}$ X. L. Luo, ${ }^{1,43}$ S. Lusso ${ }^{58 c}$ X. R. Lyu ${ }^{47}$ F. C. Ma ${ }^{31}$ H. L. Ma, ${ }^{1}$ L. L. Ma ${ }^{37}$ M. M. Ma ${ }^{1,47}$ Q. M. Ma, ${ }^{1}$ X. N. Ma,${ }^{34}$ X. X. Ma, ${ }^{1,47}$ X. Y. Ma ${ }^{1,43}$ Y. M. Ma ${ }^{37}$ F. E. Maas,${ }^{15}$ M. Maggiora, ${ }^{58 a, 58 c}$ S. Maldaner ${ }^{26}$ S. Malde ${ }^{53}$ Q. A. Malik,${ }^{57}$ A. Mangoni, ${ }^{23 b}$ Y. J. Mao, ${ }^{35}$ Z. P. Mao, ${ }^{1}$ S. Marcello, ${ }^{58 a, 58 c}$ Z. X. Meng, ${ }^{49}$ J. G. Messchendorp, ${ }^{29}$ G. Mezzadri, ${ }^{24 a}$ J. Min, ${ }^{1,43}$ T. J. Min, ${ }^{33}$ R. E. Mitchell, ${ }^{22}$ X. H. Mo, ${ }^{1,43,47}$ Y. J. Mo, ${ }^{6}$ C. Morales Morales, ${ }^{15}$ N. Yu. Muchnoi, ${ }^{10, d}$ H. Muramatsu, ${ }^{51}$ A. Mustafa, ${ }^{4}$ S. Nakhoul, ${ }^{11, \mathrm{~g}}$ Y. Nefedov ${ }^{27}$ F. Nerling, ${ }^{11, \mathrm{~g}}$ I. B. Nikolaev, ${ }^{1, \mathrm{~d}}$ Z. Ning, ${ }^{1,43}$ S. Nisar, ${ }^{8, \mathrm{k}}$ S. L. Niu, ${ }^{1,43}$ S. L. Olsen, ${ }^{47}$ Q. Ouyang, ${ }^{1,43,47}$ S. Pacetti, ${ }^{23 b}$ Y. Pan,${ }^{55,43}$ M. Papenbrock,${ }^{59}$ P. Patteri, ${ }^{23 a}$ M. Pelizaeus, ${ }^{4}$ H. P. Peng, ${ }^{55,43}$ K. Peters, ${ }^{11, g}$ J. Pettersson, ${ }^{59}$ J. L. Ping, ${ }^{32}$ R. G. Ping, ${ }^{1,47}$ A. Pitka, ${ }^{4}$ R. Poling, ${ }^{51}$ V. Prasad ${ }^{55,43}$ M. Qi,${ }^{33}$ T. Y. Qi,${ }^{2}$ S. Qian, ${ }^{1,43}$ C. F. Qiao, ${ }^{47}$ N. Qin, ${ }^{60}$ X. P. Qin, ${ }^{13}$ X. S. Qin, ${ }^{4}$ Z. H. Qin, ${ }^{1,43}$ J. F. Qiu, ${ }^{1}$ S. Q. Qu ${ }^{34}$ K. H. Rashid, ${ }^{57, i}$ C. F. Redmer ${ }^{26}$ M. Richter, ${ }^{4}$ M. Ripka, ${ }^{26}$ A. Rivetti, ${ }^{58 \mathrm{c}}$ V. Rodin, ${ }^{29}$ M. Rolo ${ }^{58 \mathrm{c}}$ G. Rong, ${ }^{1,47}$ Ch. Rosner, ${ }^{15}$ M. Rump ${ }^{52}$ A. Sarantsev,${ }^{27, \mathrm{e}}$ M. Savrié, ${ }^{24 \mathrm{~b}}$

K. Schoenning $\odot,{ }^{59}$ W. Shan, ${ }^{19}$ X. Y. Shan, ${ }^{55,43}$ M. Shao,${ }^{55,43}$ C. P. Shen, ${ }^{2}$ P. X. Shen ${ }^{34}$ X. Y. Shen, ${ }^{1,47}$ H. Y. Sheng, ${ }^{1}$ X. Shi, ${ }^{1,43}$ X. D. Shi,${ }^{55,43}$ J. J. Song, ${ }^{37}$ Q. Q. Song, ${ }^{55,43}$ X. Y. Song, ${ }^{1}$ S. Sosio, ${ }^{58 a, 58 c}$ C. Sowa, ${ }^{4}$ S. Spataro,${ }^{58 a, 58 c}$ F. F. Sui, ${ }^{37}$ G. X. Sun, ${ }^{1}$ J. F. Sun ${ }^{16}$ L. Sun, ${ }^{60}$ S. S. Sun,${ }^{1,47}$ X. H. Sun, ${ }^{1}$ Y. J. Sun,${ }^{55,43}$ Y. K. Sun,${ }^{55,43}$ Y. Z. Sun, ${ }^{1}$ Z. J. Sun, ${ }^{1,43}$ Z. T. Sun, ${ }^{1}$ Y. T. Tan,${ }^{55,43}$ C. J. Tang, ${ }^{40}$ G. Y. Tang, ${ }^{1}$ X. Tang,,${ }^{1}$ V. Thoren, ${ }^{59}$ B. Tsednee, ${ }^{25}$ I. Uman, ${ }^{46 d}$ B. Wang, ${ }^{1}$ B. L. Wang,${ }^{47}$

C. W. Wang, ${ }^{33}$ D. Y. Wang, ${ }^{35}$ H. H. Wang, ${ }^{37}$ K. Wang, ${ }^{1,43}$ L. L. Wang, ${ }^{1}$ L. S. Wang, ${ }^{1}$ M. Wang, ${ }^{37}$ M. Z. Wang, ${ }^{35}$

Meng Wang, ${ }^{1,47}$ P. L. Wang, ${ }^{1}$ R. M. Wang, ${ }^{61}$ W. P. Wang, ${ }^{55,43}$ X. Wang, ${ }^{35}$ X. F. Wang, ${ }^{1}$ X. L. Wang, ${ }^{9, j}$ Y. Wang, ${ }^{44}$ Y. Wang, ${ }^{55,43}$ Y. F. Wang, ${ }^{1,43,47}$ Z. Wang, ${ }^{1,43}$ Z. G. Wang, ${ }^{1,43}$ Z. Y. Wang, ${ }^{1}$ Zongyuan Wang, ${ }^{1,47}$ T. Weber, ${ }^{4}$ D. H. Wei, ${ }^{12}$ P. Weidenkaff, ${ }^{26}$ H. W. Wen, ${ }^{32}$ S. P. Wen, ${ }^{1}$ U. Wiedner, ${ }^{4}$ G. Wilkinson, ${ }^{53}$ M. Wolke, ${ }^{59}$ L. H. Wu, ${ }^{1}$ L. J. Wu, ${ }^{1,47}$ Z. Wu, ${ }^{1,43}$ L. Xia, ${ }^{55,43}$ Y. Xia, ${ }^{20}$ S. Y. Xiao, ${ }^{1}$ Y. J. Xiao, ${ }^{1,47}$ Z. J. Xiao, ${ }^{32}$ Y. G. Xie, ${ }^{1,43}$ Y. H. Xie, ${ }^{6}$ T. Y. Xing,,${ }^{1,47}$ X. A. Xiong,,${ }^{1,47}$ Q. L. Xiu, ${ }^{1,43}$ G. F. Xu, ${ }^{1}$ J. J. Xu, ${ }^{33}$ L. Xu, ${ }^{1}$ Q. J. Xu, ${ }^{14}$ W. Xu, ${ }^{1,47}$ X. P. Xu ${ }^{41}$ F. Yan, ${ }^{56}$ L. Yan, ${ }^{58 a, 58 c}$ W. B. Yan, ${ }^{55,43}$ W. C. Yan, ${ }^{2}$ Y. H. Yan, ${ }^{20}$ H. J. Yang, ${ }^{38, h}$ H. X. Yang, ${ }^{1}$ L. Yang, ${ }^{60}$ R. X. Yang, ${ }^{55,43}$ S. L. Yang, ${ }^{1,47}$ Y. H. Yang, ${ }^{33}$ Y. X. Yang, ${ }^{12}$ Yifan Yang, ${ }^{1,47}$ Z. Q. Yang, ${ }^{20}$ M. Ye ${ }^{1,43}$ M. H. Ye, ${ }^{7}$ J. H. Yin, ${ }^{1}$ Z. Y. You, ${ }^{44}$ B. X. Yu, ${ }^{1,43,47}$ C. X. Yu, ${ }^{34}$ J. S. Yu, ${ }^{20}$ C. Z. Yuan, ${ }^{1,47}$ X. Q. Yuan, ${ }^{35}$ Y. Yuan, ${ }^{1}$ A. Yuncu, ${ }^{46 b, a}$ A. A. Zafar, ${ }^{57}$ Y. Zeng, ${ }^{20}$ B. X. Zhang, ${ }^{1}$ B. Y. Zhang, ${ }^{1,43}$ C. C. Zhang, ${ }^{1}$ D. H. Zhang, ${ }^{1}$ H. H. Zhang, ${ }^{44}$ H. Y. Zhang, ${ }^{1,43}$ J. Zhang, ${ }^{1,47}$ J. L. Zhang, ${ }^{61}$ J. Q. Zhang, ${ }^{4}$ J. W. Zhang, ${ }^{1,43,47}$ J. Y. Zhang, ${ }^{1}$ 
J. Z. Zhang, ${ }^{1,47}$ K. Zhang, ${ }^{1,47}$ L. Zhang, ${ }^{45}$ S. F. Zhang, ${ }^{33}$ T. J. Zhang, ${ }^{38, h}$ X. Y. Zhang, ${ }^{37}$ Y. Zhang, ${ }^{55,43}$ Y. H. Zhang, ${ }^{1,43}$ Y. T. Zhang, ${ }^{55,43}$ Yang Zhang, ${ }^{1}$ Yao Zhang, ${ }^{1}$ Yi Zhang, ${ }^{9, j}$ Yu Zhang, ${ }^{47}$ Z. H. Zhang, ${ }^{6}$ Z. P. Zhang, ${ }^{55}$ Z. Y. Zhang, ${ }^{60}$ G. Zhao, ${ }^{1}$ J. W. Zhao, ${ }^{1,43}$ J. Y. Zhao, ${ }^{1,47}$ J. Z. Zhao, ${ }^{1,43}$ Lei Zhao, ${ }^{55,43}$ Ling Zhao, ${ }^{1}$ M. G. Zhao, ${ }^{34}$ Q. Zhao, ${ }^{1}$ S. J. Zhao, ${ }^{63}$ T. C. Zhao, ${ }^{1}$ Y. B. Zhao, ${ }^{1,43}$ Z. G. Zhao, ${ }^{55,43}$ A. Zhemchugov, ${ }^{27, b}$ B. Zheng, ${ }^{56}$ J. P. Zheng, ${ }^{1,43}$ Y. Zheng, ${ }^{35}$ Y. H. Zheng, ${ }^{47}$ B. Zhong, ${ }^{32}$ L. Zhou, ${ }^{1,43}$ L. P. Zhou, ${ }^{1,47}$ Q. Zhou, ${ }^{1,47}$ X. Zhou, ${ }^{60}$ X. K. Zhou, ${ }^{47}$ X. R. Zhou ${ }^{55,43}$ Xiaoyu Zhou, ${ }^{20}$ Xu Zhou, ${ }^{20}$ A. N. Zhu, ${ }^{1,47}$ J. Zhu, ${ }^{34}$ J. Zhu, ${ }^{44}$ K. Zhu, ${ }^{1}$ K. J. Zhu, ${ }^{1,43,47}$ S. H. Zhu, ${ }^{54}$ W. J. Zhu, ${ }^{34}$ X. L. Zhu, ${ }^{45}$ Y. C. Zhu, ${ }^{55,43}$ Y. S. Zhu, ${ }^{1,47}$ Z. A. Zhu, ${ }^{1,47}$ J. Zhuang, ${ }^{1,43}$ B.S. Zou, ${ }^{1}$ and J. H. Zou ${ }^{1}$

\title{
(BESIII Collaboration)
}

\author{
${ }^{1}$ Institute of High Energy Physics, Beijing 100049, People's Republic of China \\ ${ }^{2}$ Beihang University, Beijing 100191, People's Republic of China \\ ${ }^{3}$ Beijing Institute of Petrochemical Technology, Beijing 102617, People's Republic of China \\ ${ }^{4}$ Bochum Ruhr-University, D-44780 Bochum, Germany \\ ${ }^{5}$ Carnegie Mellon University, Pittsburgh, Pennsylvania 15213, USA \\ ${ }^{6}$ Central China Normal University, Wuhan 430079, People's Republic of China \\ ${ }^{7}$ China Center of Advanced Science and Technology, Beijing 100190, People's Republic of China \\ ${ }^{8}$ COMSATS University Islamabad, Lahore Campus, Defence Road, Off Raiwind Road, 54000 Lahore, Pakistan \\ ${ }^{9}$ Fudan University, Shanghai 200443, People's Republic of China \\ ${ }^{10}$ G.I. Budker Institute of Nuclear Physics SB RAS (BINP), Novosibirsk 630090, Russia \\ ${ }^{11}$ GSI Helmholtzcentre for Heavy Ion Research GmbH, D-64291 Darmstadt, Germany \\ ${ }^{12}$ Guangxi Normal University, Guilin 541004, People's Republic of China \\ ${ }^{13}$ Guangxi University, Nanning 530004, People's Republic of China \\ ${ }^{14}$ Hangzhou Normal University, Hangzhou 310036, People's Republic of China \\ ${ }^{15}$ Helmholtz Institute Mainz, Johann-Joachim-Becher-Weg 45, D-55099 Mainz, Germany \\ ${ }^{16}$ Henan Normal University, Xinxiang 453007, People's Republic of China \\ ${ }^{17}$ Henan University of Science and Technology, Luoyang 471003, People's Republic of China \\ ${ }^{18}$ Huangshan College, Huangshan 245000, People's Republic of China \\ ${ }^{19}$ Hunan Normal University, Changsha 410081, People's Republic of China \\ ${ }^{20}$ Hunan University, Changsha 410082, People's Republic of China \\ ${ }^{21}$ Indian Institute of Technology Madras, Chennai 600036, India \\ ${ }^{22}$ Indiana University, Bloomington, Indiana 47405, USA \\ ${ }^{23 a}$ INFN Laboratori Nazionali di Frascati, I-00044 Frascati, Italy \\ ${ }^{23 \mathrm{~b}}$ INFN and University of Perugia, I-06100 Perugia, Italy \\ ${ }^{24 a}$ INFN Sezione di Ferrara, I-44122 Ferrara, Italy \\ ${ }^{24 \mathrm{~b}}$ University of Ferrara, I-44122 Ferrara, Italy \\ ${ }^{25}$ Institute of Physics and Technology, Peace Avenue 54B, Ulaanbaatar 13330, Mongolia \\ ${ }^{26}$ Johannes Gutenberg University of Mainz, Johann-Joachim-Becher-Weg 45, D-55099 Mainz, Germany \\ ${ }^{27}$ Joint Institute for Nuclear Research, 141980 Dubna, Moscow region, Russia \\ ${ }^{28}$ Justus-Liebig-Universitaet Giessen, II. Physikalisches Institut, Heinrich-Buff-Ring 16, D-35392 Giessen, Germany \\ ${ }^{29}$ KVI-CART, University of Groningen, NL-9747 AA Groningen, Netherlands \\ ${ }^{30}$ Lanzhou University, Lanzhou 730000, People's Republic of China \\ ${ }^{31}$ Liaoning University, Shenyang 110036, People's Republic of China \\ ${ }^{32}$ Nanjing Normal University, Nanjing 210023, People's Republic of China \\ ${ }^{33}$ Nanjing University, Nanjing 210093, People's Republic of China \\ ${ }^{34}$ Nankai University, Tianjin 300071, People's Republic of China \\ ${ }^{35}$ Peking University, Beijing 100871, People's Republic of China \\ ${ }^{36}$ Shandong Normal University, Jinan 250014, People's Republic of China \\ ${ }^{37}$ Shandong University, Jinan 250100, People's Republic of China \\ ${ }^{38}$ Shanghai Jiao Tong University, Shanghai 200240, People's Republic of China \\ ${ }^{39}$ Shanxi University, Taiyuan 030006, People's Republic of China \\ ${ }^{40}$ Sichuan University, Chengdu 610064, People's Republic of China \\ ${ }^{41}$ Soochow University, Suzhou 215006, People's Republic of China \\ ${ }^{42}$ Southeast University, Nanjing 211100, People's Republic of China \\ ${ }^{43}$ State Key Laboratory of Particle Detection and Electronics, Beijing 100049, Hefei 230026, People's Republic of China \\ ${ }^{44}$ Sun Yat-Sen University, Guangzhou 510275, People's Republic of China \\ ${ }^{45}$ Tsinghua University, Beijing 100084, People's Republic of China \\ ${ }_{46 a}$ Ankara University, 06100 Tandogan, Ankara, Turkey \\ ${ }^{46 \mathrm{~b}}$ Istanbul Bilgi University, 34060 Eyup, Istanbul, Turkey
}




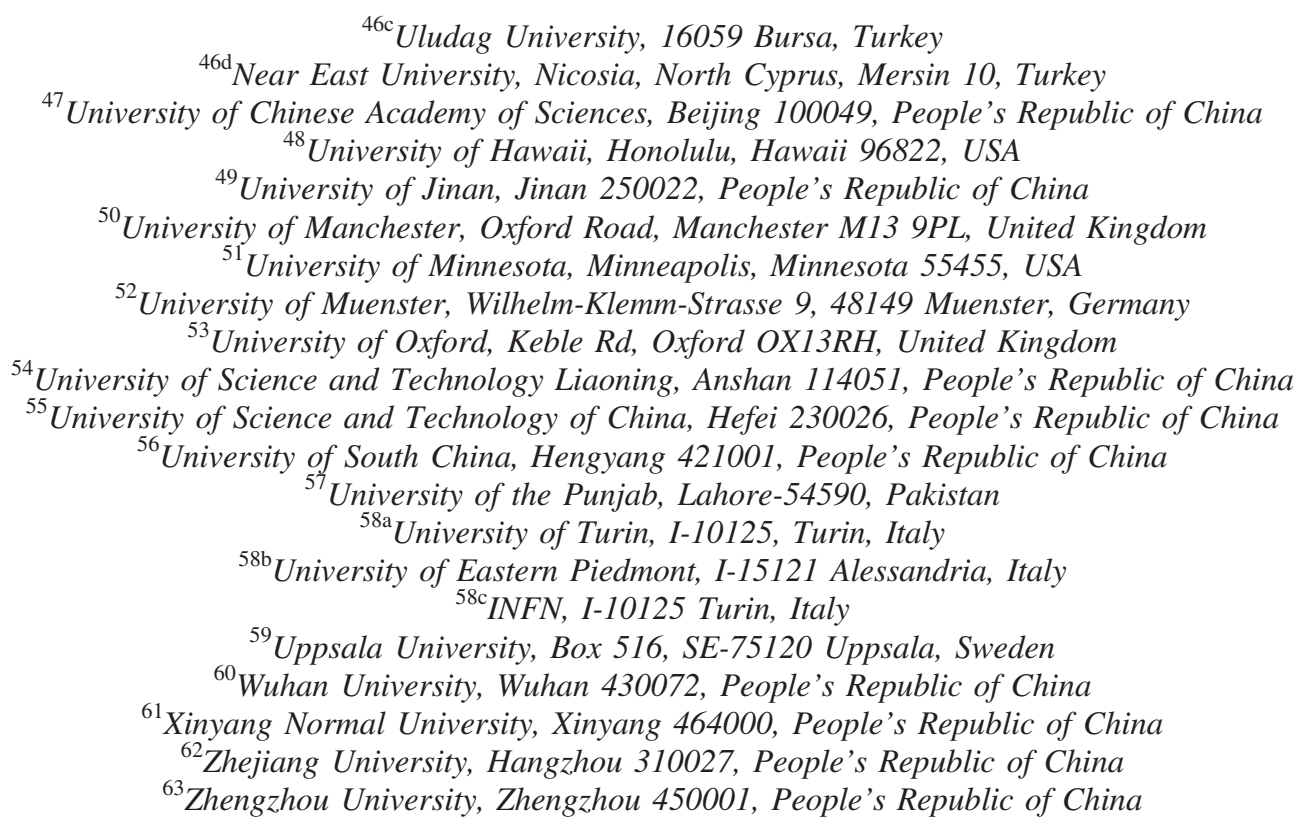

(Received 25 March 2019; revised manuscript received 26 June 2019; published 20 September 2019)

\begin{abstract}
The exclusive process $e^{+} e^{-} \rightarrow \Lambda \bar{\Lambda}$, with $\Lambda \rightarrow p \pi^{-}$and $\bar{\Lambda} \rightarrow \bar{p} \pi^{+}$, has been studied at $\sqrt{s}=2.396 \mathrm{GeV}$ for measurement of the timelike $\Lambda$ electric and magnetic form factors, $G_{E}$ and $G_{M}$. A data sample, corresponding to an integrated luminosity of $66.9 \mathrm{pb}^{-1}$, was collected with the BESIII detector for this purpose. A multidimensional analysis with a complete decomposition of the spin structure of the reaction enables a determination of the modulus of the ratio $R=\left|G_{E} / G_{M}\right|$ and, for the first time for any baryon, the relative phase $\Delta \Phi=\Phi_{E}-\Phi_{M}$. The resulting values are $R=0.96 \pm 0.14$ (stat) \pm 0.02 (syst) and $\Delta \Phi=37^{\circ} \pm 12^{\circ}$ (stat) $\pm 6^{\circ}$ (syst), respectively. These are obtained using the recently established and most precise value of the asymmetry parameter $\alpha_{\Lambda}=0.750 \pm 0.010$ measured by BESIII. In addition, the cross section is measured with unprecedented precision to be $\sigma=118.7 \pm 5.3$ (stat) \pm 5.1 (syst) pb, which corresponds to an effective form factor of $|G|=0.123 \pm 0.003$ (stat) \pm 0.003 (syst). The contribution from two-photon exchange is found to be negligible. Our result enables the first complete determination of baryon timelike electromagnetic form factors.
\end{abstract}

DOI: 10.1103/PhysRevLett.123.122003

One of the most challenging questions in contemporary physics is to understand the strong interaction in the confinement domain, i.e., where quarks form hadrons. This puzzle manifests itself in one of the most abundant building blocks of the Universe: the nucleon. Neither its size [1], its spin [2], nor its intrinsic structure [3] is fully understood. The latter has been extracted from spacelike electromagnetic form factors (EMFFs), fundamental properties of hadrons that have been studied since the 1960s [4]. In particular, the neutron charge distribution is intriguing [3]. Hyperons provide a new angle on the nucleon puzzle:

Published by the American Physical Society under the terms of the Creative Commons Attribution 4.0 International license. Further distribution of this work must maintain attribution to the author(s) and the published article's title, journal citation, and DOI. Funded by SCOAP ${ }^{3}$.
What happens if we replace one of the $u$ and $d$ quarks with a heavier $s$ quark? A systematic comparison of octet baryons sheds light on to what extent $\mathrm{SU}(3)$ flavor symmetry is broken. The importance of hyperon structure was pointed out as early as 1960 [5], but has not been subjected to rigorous experimental studies until now. The main reason is that spacelike EMFFs of hyperons are not straightforward to access experimentally since their finite lifetime makes them unsuitable as beams and targets. Instead, the electromagnetic structure can be quantified in terms of timelike form factors. These can be accessed in, e.g., hyperon-antihyperon production in $e^{+} e^{-} \rightarrow \gamma^{*} \rightarrow Y \bar{Y}$, where $Y$ denotes the hyperon. The experimentally accessible timelike form factors are related to more intuitive spacelike quantities such as charge and magnetization densities by dispersion relations [6].

Spin $1 / 2$ baryons are described by two independent EMFFs, commonly the electric form factor $G_{E}$ and the 
magnetic form factor $G_{M}$. These are functions of the fourmomentum transfer squared, $s=q^{2}: G_{E} \equiv G_{E}(s)$ and $G_{M} \equiv G_{M}(s)$. As a consequence of the optical theorem, timelike EMFFs above the two-pion threshold $s \geq 4 m_{\pi}^{2}$ have a nonzero imaginary part. This means that if $G_{E}$ and $G_{M}$ are different, they have a nonzero relative phase [7,8]. This phase, $\Delta \Phi \equiv \Delta \Phi(s)$, must be zero at the kinematic threshold, where by definition the electric and the magnetic form factors are equal.

The asymptotic behavior of the timelike EMFFs as $s \rightarrow \infty$ can be obtained from the corresponding spacelike region as a consequence of the Phragmén-Lindelöf theorem [9]. Since in the spacelike region, EMFFs are real, the same must be true for the timelike region in the $s \rightarrow \infty$ limit. This means that the phase tends to integer multiples of $\pi$ radians, depending on the $s$ power-law behavior $[10,11]$ and the eventual presence of spacelike zeros [8]. However, for intermediate $s$ the phase can have any value. This would introduce polarization effects on the final state, even if the initial state is unpolarized [7]. Thanks to the weak, parity violating decays of hyperons, the polarization is experimentally accessible. This provides unique opportunities compared to nucleons.

The recent development of high-intensity electron-positron colliders in the strange and charm energy region offers new possibilities for detailed structure studies of hyperons. The first measurement of $e^{+} e^{-} \rightarrow \Lambda \bar{\Lambda}$ production was reported by the DM2 Collaboration [12]. The first determination of the $\Lambda$ EMFFs was provided by the $B A B A R$ Collaboration, using the initial state radiation (ISR) method [13]. However, the sample was insufficient for a clear separation of the electric and magnetic form factors. An attempt was made to extract the phase from the $\Lambda$ polarization, but the result was inconclusive [13]. The cross section of the production of protons and ground-state hyperons in $e^{+} e^{-}$annihilations at $\sqrt{s}=3.69,3.77$, and $4.17 \mathrm{GeV}$ was measured with CLEO-c data. The magnetic form factors were extracted assuming $\left|G_{E}\right|=\left|G_{M}\right|$ [14]. The BESIII Collaboration performed in 2011-2012 an energy scan, enabling an investigation of the $\Lambda$ production cross section at four energies between $\sqrt{s}=2.23$ and $\sqrt{s}=3.08 \mathrm{GeV}$. An unexpected enhancement at the kinematic threshold was observed [15]. At higher energies, the statistical precision was improved compared to previous experiments, though still not sufficient to extract the form factor ratio $R \equiv\left|G_{E} / G_{M}\right|$. The recent experimental progress has resulted in an increasing interest from the theory community. For instance, predictions of the relative phase have been made, based on various $\Lambda \bar{\Lambda}$ potential models [16] with input data from the PS185 experiment [17].

In this Letter, the exclusive process $e^{+} e^{-} \rightarrow \Lambda \bar{\Lambda}$ $\left(\Lambda \rightarrow p \pi^{-}, \bar{\Lambda} \rightarrow \bar{p} \pi^{+}\right)$is studied at $\sqrt{s}=2.396 \mathrm{GeV}$. This energy gives the optimal $\Lambda \bar{\Lambda}$ detection rate-at larger energies, the cross section becomes too small [13], and at lower energies, the reconstruction efficiency goes down rapidly. The latter is due to the pions from the $\Lambda$ decays that have too low momenta to reach the detectors [15]. In the following, we present our measurements of the cross section $\sigma \equiv \sigma(s)$, the ratio $R=\left|G_{E} / G_{M}\right|$, and, for the first time, the relative phase $\Delta \Phi$.

Assuming one-photon exchange $\left(e^{+} e^{-} \rightarrow \gamma^{*} \rightarrow B \bar{B}\right)$, the Born cross section of spin $1 / 2$ baryon-antibaryon pair production can be parametrized in terms of $G_{E}$ and $G_{M}$ :

$$
\sigma_{B \bar{B}}(s)=\frac{4 \pi \alpha^{2} \beta}{3 s}\left(\left|G_{M}(s)\right|^{2}+\frac{1}{2 \tau}\left|G_{E}(s)\right|^{2}\right) .
$$

Here, $\alpha=1 / 137.036$ is the fine-structure constant, $\beta=$ $\sqrt{1-4 m_{B}^{2} / s}$ the velocity of the produced baryon, $m_{B}$ the mass of the baryon, and $\tau=s /\left(4 m_{B}^{2}\right)$.

In most previous experiments, where the small data samples did not allow for a separation between $G_{E}$ and $G_{M}$, the effective form factor has been studied. It is defined as

$$
|G(s)| \equiv \sqrt{\frac{\sigma_{B \bar{B}}(s)}{\left(1+\frac{1}{2 \tau}\right)\left(\frac{4 \pi \alpha^{2} \beta}{3 s}\right)}}
$$

and gives a quantitative indication of the deviation from the pointlike cross section.

A complete decomposition of the complex $G_{E}$ and $G_{M}$ requires a multidimensional analysis of the reaction and the subsequent baryon decays. In Refs. $[18,19]$, the joint decay distribution of $e^{+} e^{-} \rightarrow \Lambda \bar{\Lambda}\left(\Lambda \rightarrow p \pi^{-}, \bar{\Lambda} \rightarrow \bar{p} \pi^{+}\right)$ was derived in terms of the phase $\Delta \Phi$ and the angular distribution parameter $\eta=\left(\tau-R^{2}\right) /\left(\tau+R^{2}\right)$ :

$$
\begin{aligned}
\mathcal{W}(\boldsymbol{\xi})= & \mathcal{T}_{0}+\eta \mathcal{T}_{5}-\alpha_{\Lambda}^{2}\left[\mathcal{T}_{1}+\sqrt{1-\eta^{2}} \cos (\Delta \Phi) \mathcal{T}_{2}+\eta \mathcal{T}_{6}\right] \\
& +\alpha_{\Lambda} \sqrt{1-\eta^{2}} \sin (\Delta \Phi)\left(\mathcal{T}_{3}-\mathcal{T}_{4}\right)
\end{aligned}
$$

where $\alpha_{\Lambda}$ denotes the decay asymmetry of the $\Lambda \rightarrow p \pi^{-}$ decay. The seven functions $\mathcal{T}_{k}(\boldsymbol{\xi})$ do not depend on the parameters $\eta$ and $\Delta \Phi$, but only on the measured angles:

$\mathcal{T}_{0}(\boldsymbol{\xi})=1$,

$\mathcal{T}_{1}(\boldsymbol{\xi})=\sin ^{2} \theta \sin \theta_{1} \sin \theta_{2} \cos \phi_{1} \cos \phi_{2}+\cos ^{2} \theta \cos \theta_{1} \cos \theta_{2}$,

$\mathcal{T}_{2}(\boldsymbol{\xi})=\sin \theta \cos \theta\left(\sin \theta_{1} \cos \theta_{2} \cos \phi_{1}+\cos \theta_{1} \sin \theta_{2} \cos \phi_{2}\right)$,

$\mathcal{T}_{3}(\boldsymbol{\xi})=\sin \theta \cos \theta \sin \theta_{1} \sin \phi_{1}$,

$\mathcal{T}_{4}(\boldsymbol{\xi})=\sin \theta \cos \theta \sin \theta_{2} \sin \phi_{2}$,

$\mathcal{T}_{5}(\boldsymbol{\xi})=\cos ^{2} \theta$,

$\mathcal{T}_{6}(\boldsymbol{\xi})=\cos \theta_{1} \cos \theta_{2}-\sin ^{2} \theta \sin \theta_{1} \sin \theta_{2} \sin \phi_{1} \sin \phi_{2}$.

The five angles measured are the $\Lambda$ scattering angle $\theta$ with respect to the electron beam, the proton helicity angles $\theta_{1}$ and $\phi_{1}$ from the $\Lambda \rightarrow p \pi^{-}$decay, and the antiproton 


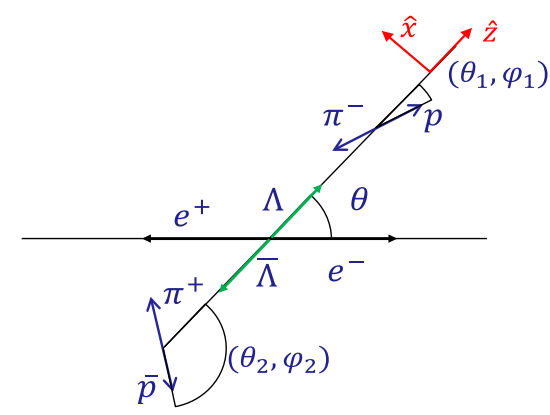

FIG. 1. Definition of the coordinate system used to describe the $e^{+} e^{-} \rightarrow \Lambda \bar{\Lambda}\left(\Lambda \rightarrow p \pi^{-}, \bar{\Lambda} \rightarrow \bar{p} \pi^{+}\right)$process.

helicity angles $\theta_{2}$ and $\phi_{2}$ from the $\bar{\Lambda} \rightarrow \bar{p} \pi^{+}$decay. The decay angles are defined in the rest system of the $\Lambda$ and the $\bar{\Lambda}$, respectively. We define a right-handed system where the $z$ axis is oriented along the $\Lambda$ momentum $\mathbf{p}_{\Lambda}=-\mathbf{p}_{\bar{\Lambda}}$ in the $e^{+} e^{-}$rest system. The $y$ axis is perpendicular to the reaction plane and is oriented along the $\mathbf{k}_{e^{-}} \times \mathbf{p}_{\Lambda}$ direction, where $\mathbf{k}_{e^{-}}=-\mathbf{k}_{e^{+}}$is the electron beam momentum in the $e^{+} e^{-}$rest system. The definitions of the angles are illustrated in Fig. 1.

The term $\mathcal{T}_{0}+\eta \mathcal{T}_{5}$ in Eq. (3) describes the scattering angle distribution of the $\Lambda$ hyperon. The term $\alpha_{\Lambda} \sqrt{1-\eta^{2}} \sin (\Delta \Phi)\left(\mathcal{T}_{3}-\mathcal{T}_{4}\right)$ accounts for the transverse polarization $P_{y}$ of the $\Lambda$ and $\bar{\Lambda}$. In particular, the $\Lambda$ transverse polarization $P_{y}$ is given by

$$
P_{y}=\frac{\sqrt{1-\eta^{2}} \sin \theta \cos \theta}{1+\eta \cos ^{2} \theta} \sin (\Delta \Phi) .
$$

Finally, the $\alpha_{\Lambda}^{2}\left[\mathcal{T}_{1}+\sqrt{1-\eta^{2}} \cos (\Delta \Phi) \mathcal{T}_{2}+\eta \mathcal{T}_{6}\right]$ term describes the spin correlations between the two hyperons.

The asymmetry parameter $\alpha_{\Lambda}$ has recently been measured by the BESIII Collaboration to be $\alpha_{\Lambda}=$ $0.750 \pm 0.010$ [20]. This value has been adopted by the Particle Data Group in the 2019 update of Ref. [21].

A data sample corresponding to an integrated luminosity of $66.9 \mathrm{pb}^{-1}$ was collected with the Beijing spectrometer (BESIII) at the Beijing Electron Positron Collider (BEPCII). The BESIII detector has a geometrical acceptance of $93 \%$ of the solid angle. BESIII contains a small-cell, helium-based main drift chamber (MDC), a time-of-flight system based on plastic scintillators, an electromagnetic calorimeter made of $\mathrm{CsI}(\mathrm{Tl})$ crystals, a muon counter made of resistive plate chambers, and a superconducting solenoid magnet with a central field of 1.0 T. A detailed description of the detector and its performance can be found in Ref. [22].

The particle propagation through the detector is modeled using a GEANT-based [23] Monte Carlo (MC) simulation software package, BOost [24]. The multidimensional analysis for determination of $R$ and $\Delta \Phi$ enables a modelindependent efficiency correction. The simulations for this purpose are performed with a MC sample generated by a phase space generator. The determination of $\sigma$ and $G$ was found to be more precise using an approach with a global efficiency. The latter was obtained from simulations of $e^{+} e^{-} \rightarrow \Lambda \bar{\Lambda}\left(\Lambda \rightarrow p \pi^{-}, \bar{\Lambda} \rightarrow \bar{p} \pi^{+}\right)$using the measured values of $\left|G_{E} / G_{M}\right|$ as input to the CONEXC generator [25]. In CONEXC, higher order processes with one radiative photon are taken into account. For background studies, an inclusive MC sample of continuum processes $e^{+} e^{-} \rightarrow q \bar{q}$ with $q=u, d, s$ is used.

In the analysis, events are reconstructed by the final state particles $p, \pi^{-}, \bar{p}$, and $\pi^{+}$. We therefore require at least four charged tracks per event. Each track must be reconstructed within the MDC, i.e., with polar angles $\theta$ fulfilling $|\cos \theta|<0.93$, measured in the laboratory frame between the direction of the track and the direction of the $e^{+}$beam. The momentum of each track must be smaller than $0.5 \mathrm{GeV} / c$. It was found in simulations that the momentum distributions of pions and protons or antiprotons are well separated due to kinematics. Therefore, we can identify tracks with momenta smaller than $0.2 \mathrm{GeV} / c$ as $\pi^{+} / \pi^{-}$ candidates, whereas tracks with momenta larger than $0.2 \mathrm{GeV} / c$ are identified as $p / \bar{p}$ candidates. This was found to give an optimal signal-to-background ratio and the results are consistent with those obtained using standard particle identification criteria.

The $\Lambda$ and $\bar{\Lambda}$ candidates are reconstructed by fitting each $p \pi^{-}\left(\bar{p} \pi^{+}\right)$to a common vertex corresponding to the decay of $\Lambda(\bar{\Lambda})$. A four-constraint (4C) kinematic fit is applied on the $\Lambda$ and $\bar{\Lambda}$ candidates, using energy and momentum conservation in $e^{+} e^{-} \rightarrow \Lambda \bar{\Lambda}$ and requiring $\chi_{4 C}^{2}<50$. We require the $p \pi^{-} / \bar{p} \pi^{+}$invariant mass to fulfill $\left|M\left(p \pi^{-} / \bar{p} \pi^{+}\right)-m_{\Lambda}\right|<6 \mathrm{MeV} / c^{2}$. The $M\left(p \pi^{-}\right)$distribution is shown in Fig. 2. Here, $m_{\Lambda}$ is the nominal mass of $\Lambda$ from the Particle Data Group [21]. The mass window corresponds to $\pm 4 \sigma$ of $\left|M\left(p \pi^{-} / \bar{p} \pi^{+}\right)\right|$mass resolution.

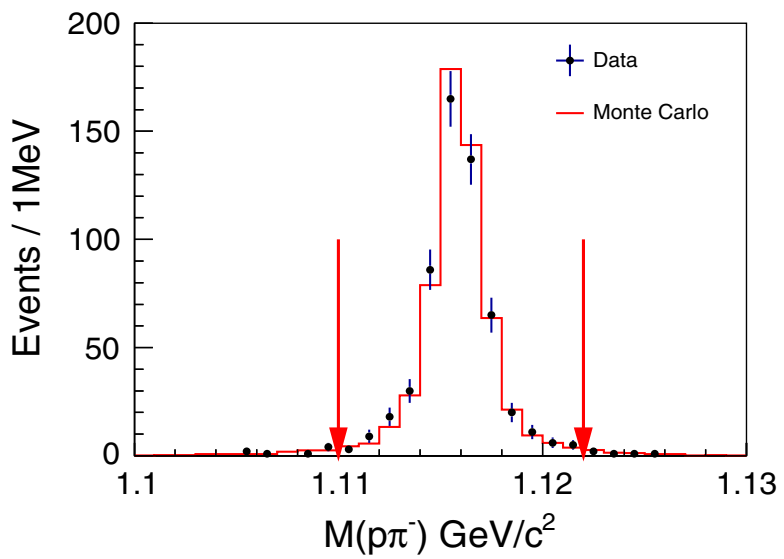

FIG. 2. The invariant mass of $p \pi^{-}$for BESIII data (black dots) and Monte Carlo data (red line) fulfilling all selection criteria except those on invariant mass. The MC data are normalized to the total number of events in the data. 

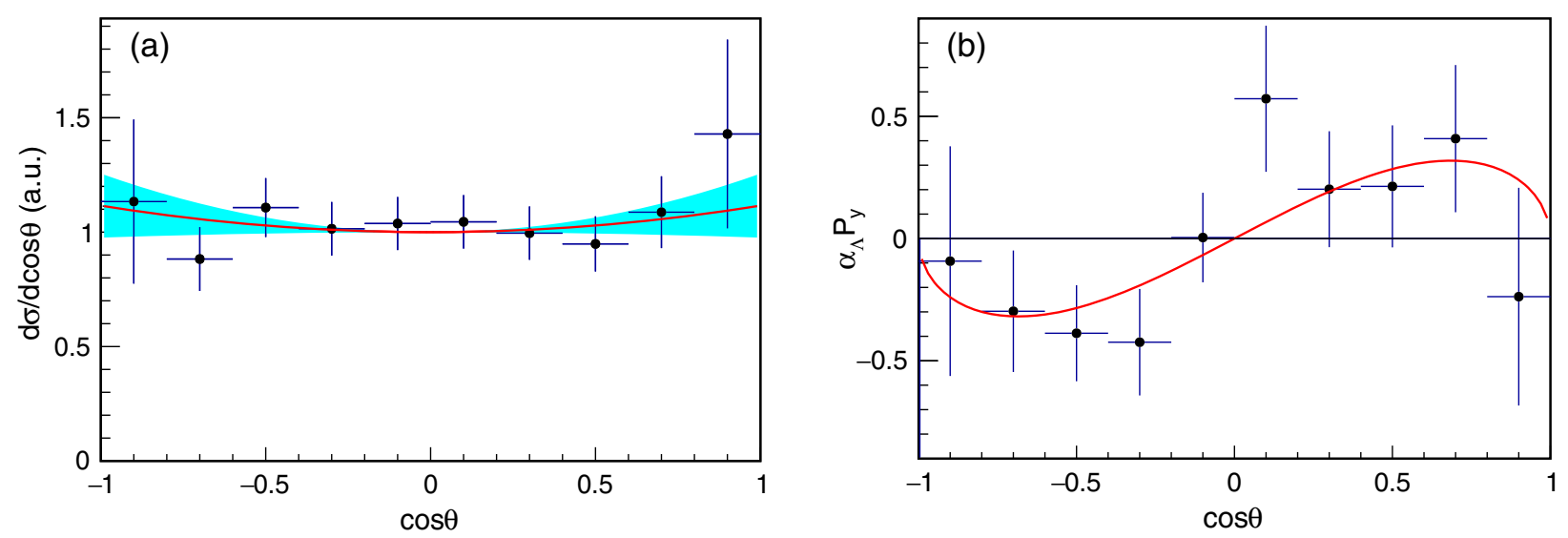

FIG. 3. (a) The acceptance corrected $\Lambda$ scattering angle distribution. The experimental distribution (points) is normalized to yield $A=1$ obtained fitting $A+B \cos ^{2} \theta$ to the data. The red line is $1+\eta \cos ^{2} \theta$ with $\eta=0.12$ and the band corresponds to the statistical uncertainty. (b) The product of $\alpha_{\Lambda}$ and $\Lambda$ polarization $P_{y}$ as a function of the scattering angle. The dots are the data, the red line the polarization corresponding to the $\Delta \Phi$ and $\eta$ obtained in the maximum log-likelihood fit described in the text. The data in these plots have been efficiency corrected using a multidimensional method using MC simulations with parameters determined from the maximum loglikelihood fit.

After applying all event selection criteria, 555 event candidates remain in our data sample.

The background channels are identified by performing inclusive $q \bar{q}$ simulations. The main contribution are events from $\Delta^{++} \bar{p} \pi^{-}\left(\bar{\Delta}^{--} p \pi^{+}\right)$and nonresonant $p \bar{p} \pi^{+} \pi^{-}$production, i.e., reactions with similar topology as $e^{+} e^{-} \rightarrow$ $\Lambda \bar{\Lambda}\left(\Lambda \rightarrow p \pi^{-}, \bar{\Lambda} \rightarrow \bar{p} \pi^{+}\right)$. The contamination is found to be on the percent level. A two-dimensional sideband study provides an independent, data-driven method to quantify the background contribution from events with misidentified $\Lambda / \bar{\Lambda}$. The $\Lambda / \bar{\Lambda}$ sideband regions are defined within $1.097<M\left(p \pi^{-} / \bar{p} \pi^{+}\right)<1.109 \mathrm{GeV} / c^{2}$ and $1.123<M\left(p \pi^{-} / \bar{p} \pi^{+}\right)<1.135 \mathrm{GeV} / c^{2}$ for events with a $\bar{\Lambda} / \Lambda$ candidate. The number of background events is determined to be $14 \pm 4$, corresponding to a background level of $2.5 \%$.

In our analysis, we extract the parameters $\eta$ and $\Delta \Phi$ by applying a multidimensional event-by-event maximum log-likelihood fit in MINUIT [26]. The multidimensional approach takes the reconstruction efficiency into account in a model-independent way. The results from the fit are $\eta=0.12 \pm 0.14$, giving $R=0.96 \pm 0.14$, and $\Delta \Phi=$ $37^{\circ} \pm 12^{\circ}$, where the uncertainties are statistical. The correlation coefficient between $\eta$ and $\Delta \Phi$ is 0.17 . The $\Lambda$ angular distribution and the polarization, multiplied with the constant $\alpha_{\Lambda}$, as a function of the scattering angle are shown in Fig. 3. The characteristic dependence of the polarization on $\cos \theta$ is a consequence of the nonzero phase.

A thorough investigation of possible sources of systematic uncertainties has been performed. The uncertainties from the luminosity measurement, tracking, and background are found to be negligible. The non-negligible contributions from the angular fit range (for $R$ ), from requirements on $\chi_{4 C}^{2}$ (for $\Delta \Phi$ ), and requirements on the invariant mass are summarized in Table I. The total systematic uncertainty is about 7 times smaller than the statistical for $R$ and about 2 times smaller for $\Delta \Phi$.

The formalism presented in Eq. (3) assumes the onephoton exchange to be dominant in the production mechanism. A significant contribution of two-photon exchange of the lowest order results in an additional term $\kappa \cos \theta \sin ^{2} \theta$ in Eq. (3) due to interference of the one- and two-photon amplitudes [27]. This would give rise to a nonzero asymmetry in the $\Lambda$ angular distribution [28]:

$$
A=\frac{N(\cos \theta>0)-N(\cos \theta<0)}{N(\cos \theta>0)+N(\cos \theta<0)},
$$

where $A$ is related to $\kappa$ according to

$$
A=\frac{3}{4} \frac{\kappa}{3+\eta} .
$$

In this work, the asymmetry is measured to be $A=$ $0.001 \pm 0.037$ and indicates a negligible contribution from two-photon exchange with respect to the statistical precision.

TABLE I. Systematic uncertainties in $R$ and $\Delta \Phi$.

\begin{tabular}{lcc}
\hline \hline Source & $R(\%)$ & $\Delta \Phi(\%)$ \\
\hline$\chi_{4 C}^{2}$ cut & $\ldots$ & 14 \\
Mass window of $p \pi$ & 0.1 & 5.5 \\
Different range of $\cos \theta$ & 2.0 & $\ldots$ \\
Total & 2.0 & 15 \\
\hline \hline
\end{tabular}


The total cross section has been calculated using

$$
\sigma_{\Lambda \bar{\Lambda}}=\frac{N_{\text {signal }}}{\mathcal{L}_{\text {int }} \epsilon(1+\delta) \mathcal{B}},
$$

where $N_{\text {signal }}=N_{\text {data }}-N_{\text {bg }}, N_{\text {data }}=555$ is the number of events in the sample after all selection criteria, $N_{\text {bg }}=$ $14 \pm 4$ the number of events in the sidebands, $\mathcal{L}_{\text {int }}$ the integrated luminosity, and $\epsilon$ the global efficiency. The radiative correction factor $1+\delta$ is determined taking ISR and vacuum polarization into account. The factor $\mathcal{B}$ is the product of the branching fractions of $\Lambda \rightarrow p \pi^{-}$and $\bar{\Lambda} \rightarrow \bar{p} \pi^{+}$, taken from Ref. [21].

The following systematic effects contribute to the uncertainty of the cross section measurement. (i) The uncertainty from the $\Lambda$ and $\bar{\Lambda}$ reconstruction is determined to be $1.1 \%$ and $2.4 \%$, respectively, using single-tag samples of $\Lambda$ and $\bar{\Lambda}$. (ii) The kinematic fit contributes with $1.7 \%$. (iii) The model dependence of the global efficiency is evaluated by changing the input $R$ with one standard deviation $( \pm 0.14)$ in the CONEXC generator. This gives an uncertainty of $2.8 \%$. The phase $\Delta \Phi$ was found to have a negligible impact on the efficiency. (iv) The uncertainty of the integrated luminosity is $1.0 \%$ [29]. The individual uncertainties are assumed to be uncorrelated and are therefore added in quadrature, which yields a total systematic uncertainty of the cross section of $4.3 \%$. The systematic uncertainty in the effective form factor $|G|$ is obtained using error propagation.

In summary, the process $e^{+} e^{-} \rightarrow \Lambda \bar{\Lambda} \quad\left(\Lambda \rightarrow p \pi^{-}\right.$, $\bar{\Lambda} \rightarrow \bar{p} \pi^{+}$) is studied with $66.9 \mathrm{pb}^{-1}$ of data collected at $2.396 \mathrm{GeV}$. The cross section and the effective form factor are obtained to be $\sigma=118.7 \pm 5.3$ (stat) \pm 5.1 (syst) $\mathrm{pb}$ and $|G|=0.123 \pm 0.003$ (stat) \pm 0.003 (syst). The effective form factor is about one half of that of the proton at the corresponding excess energy [30]. The ratio $R=\left|G_{E} / G_{M}\right|$ is determined with unprecedented precision to be $R=0.96 \pm 0.14$ (stat) \pm 0.02 (syst). The relative phase between $G_{E}$ and $G_{M}$ is determined for the first time to be $\Delta \Phi=37^{\circ} \pm 12^{\circ}$ (stat) $\pm 6^{\circ}$ (syst).

The nonzero value of the relative phase implies that the imaginary part of the electric and the magnetic form factors is different. Equivalently, this means that not only the $s$ wave but also the $d$-wave amplitude contribute to the production and their interference results in a polarized final state.

This first complete hyperon EMFF measurement is a milestone in the study of hyperon structure, where the longterm goal is to describe charge and magnetization densities for hyperons in the same way as for nucleons [3]. In order to achieve this, similar measurements must be carried out at several energies. For nucleons, the scale at which spacelike EMFFs approach the timelike EMFFs is straightforward to extract since both spacelike and timelike EMFFs are experimentally accessible. For hyperons, for which only the timelike EMFFs can be measured, the corresponding scale can instead be obtained where the phase approaches a constant value that is an integer multiple of $\pi$. For this purpose, the methods developed for this study can be applied at other energies, provided the data sample at each energy is large enough.

In addition, this measurement offers a unique and clean opportunity to learn about the $\Lambda \bar{\Lambda}$ interaction close to threshold. In a recent theory paper [16], predictions have been made using final state interaction (FSI) potentials. The latter were obtained from fits to PS185 data from the $\bar{p} p \rightarrow$ $\Lambda \bar{\Lambda}$ reaction [17]. While the sensitivity of the energy dependence of the effective form factor $|G|$ to the $\Lambda \bar{\Lambda}$ FSI potential is very small, the predictions of $R$ and, even more, $\Delta \Phi$ depend significantly on the FSI potential. Our measurement slightly favors the model I or model II potential of Ref. [31]. This illustrates the sensitivity of our data to the $\Lambda \bar{\Lambda}$ interaction.

The BESIII Collaboration thanks the staff of BEPCII and the IHEP computing center for their strong support. This work is supported in part by National Key Basic Research Program of China under Contract No. 2015CB856700; National Natural Science Foundation of China (NSFC) under Contracts No. 11235011, No. 11335008, No. 11425524, No. 11625523, No. 11635010; the Chinese Academy of Sciences (CAS) Large-Scale Scientific Facility Program; the CAS Center for Excellence in Particle Physics (CCEPP); Joint Large-Scale Scientific Facility Funds of the NSFC and CAS under Contracts No. U1332201, No. U1532257, No. U1532258; CAS under Contracts No. KJCX2-YWN29, No. KJCX2-YW-N45, No. QYZDJ-SSW-SLH003; 100 Talents Program of CAS; National 1000 Talents Program of China; INPAC and Shanghai Key Laboratory for Particle Physics and Cosmology; German Research Foundation DFG under Contracts No. Collaborative Research Center CRC 1044, No. FOR 2359; Istituto Nazionale di Fisica Nucleare, Italy; Joint Large-Scale Scientific Facility Funds of the NSFC and CAS; Koninklijke Nederlandse Akademie van Wetenschappen (KNAW) under Contract No. 530-4CDP03; Ministry of Development of Turkey under Contract No. DPT2006K120470; National Natural Science Foundation of China (NSFC) under Contract No. 11505010; National Science and Technology fund; The Swedish Research Council; The Knut and Alice Wallenberg foundation, Sweden, Contract No. 2016.0157; U.S. Department of Energy under Contracts No. DE-FG02-05ER41374, No. DE-SC-0010118, No. DE-SC-0010504, No. DE-SC-0012069; University of Groningen (RuG) and the Helmholtzzentrum fuer Schwerionenforschung GmbH (GSI), Darmstadt; WCU Program of National Research Foundation of Korea under Contract No. R32-2008-000-10155-0. 
${ }^{\mathrm{a} A l s o}$ at: Bogazici University, 34342 Istanbul, Turkey.

${ }^{\mathrm{b}}$ Also at: Moscow Institute of Physics and Technology, Moscow 141700, Russia.

${ }^{\mathrm{c}}$ Also at: Functional Electronics Laboratory, Tomsk State University, Tomsk 634050, Russia.

${ }^{\mathrm{d}}$ Also at: Novosibirsk State University, Novosibirsk 630090, Russia.

eAlso at: NRC "Kurchatov Institute," PNPI, 188300 Gatchina, Russia.

${ }^{\mathrm{f}}$ Also at: Istanbul Arel University, 34295 Istanbul, Turkey.

${ }^{\mathrm{g}}$ Also at: Goethe University Frankfurt, 60323 Frankfurt am Main, Germany.

${ }^{h}$ Also at: Key Laboratory for Particle Physics, Astrophysics and Cosmology, Ministry of Education; Shanghai Key Laboratory for Particle Physics and Cosmology; Institute of Nuclear and Particle Physics, Shanghai 200240, People's Republic of China.

${ }^{i}$ Also at: Government College Women University, Sialkot51310 Punjab, Pakistan.

${ }^{\mathrm{j}}$ Also at: Key Laboratory of Nuclear Physics and Ion-beam Application (MOE) and Institute of Modern Physics, Fudan University, Shanghai 200443, People's Republic of China. ${ }^{\mathrm{k}}$ Also at: Harvard University, Department of Physics, Cambridge, MA 02138, USA.

[1] R. Pohl et al., Nature (London) 466, 213 (2010); C. E. Carlson, Prog. Part. Nucl. Phys. 82, 59 (2015).

[2] C. A. Aidala, S. D. Bass, D. Hasch, and G. K. Mallot, Rev. Mod. Phys. 85, 655 (2013).

[3] G. A. Miller, Phys. Rev. Lett. 99, 112001 (2007).

[4] V. Punjabi, C. F. Perdrisat, and M. K. Jones, Eur. Phys. J. A 51, 79 (2015).

[5] N. Cabibbo and R. Gatto, Phys. Rev. Lett. 313, 4 (1960); Phys. Rev. 124, 1577 (1961).

[6] M. A. Belushkin, H.-W. Hammer, and U.-G. Meißner, Phys. Rev. C 75, 035202 (2007).

[7] A. Z. Dubnickova, S. Dubnička, and M. P. Rekalo, Nuovo Cimento A 109, 241 (1996).

[8] S. Pacetti, R. Baldini Ferroli, and E. Tomasi-Gustafsson, Phys. Rep. 550-551, 1 (2015).

[9] E. C. Titchmarsh, The Theory of Functions (Oxford University Press, 1939).

[10] V. A. Matveev, R. M. Muradyan, and A. N. Tavkhelidze, Theor. Mat. Fiz. 15, 332 (1973).
[11] S. Brodsky and G. R. Farrar, Phys. Rev. Lett. 31, 1153 (1973).

[12] D. Bisello et al. (DM2 Collaboration), Z. Phys. C 48, 23 (1990).

[13] B. Aubert et al. (BABAR Collaboration), Phys. Rev. D 76, 092006 (2007).

[14] S. Dobbs, A. Tomaradze, T. Xiao, K. K. Seth, and G. Bonvicini, Phys. Lett. B 739, 90 (2014); S. Dobbs, K. K. Seth, A. Tomaradze, T. Xiao, and G. Bonvicini, Phys. Rev. D 96, 092004 (2017).

[15] M. Ablikim et al. (BESIII Collaboration), Phys. Rev. D 97, 032013 (2018).

[16] J. Haidenbauer and U.-G. Meißner, Phys. Lett. B 761, 456 (2016).

[17] P. D. Barnes et al. (PS185 Collaboration), Phys. Lett. B 189, 249 (1987); 229, 432 (1989); Nucl. Phys. A526, 574 (1991); Phys. Rev. C 54, 1877 (1996).

[18] G. Fäldt, Eur. Phys. J. A 52, 141 (2016).

[19] G. Fäldt and A. Kupsc, Phys. Lett. B 772, 16 (2017).

[20] M. Ablikim et al. (BESIII Collaboration), Nat. Phys. 15, 631 (2019).

[21] M. Tanabashi et al. (Particle Data Group), Phys. Rev. D 98, 030001 (2018).

[22] M. Ablikim et al. (BESIII Collaboration), Nucl. Instrum. Methods Phys. Res., Sect. A 614, 345 (2010).

[23] S. Agostinelli et al. (GEANT4 Collaboration), Nucl. Instrum. Methods Phys. Res., Sect. A 506, 250 (2003).

[24] Z. Y. Deng et al., Chin. Phys. C 30, 371 (2006).

[25] R. G. Ping et al., Chin. Phys. C 38, 083001 (2014).

[26] J. Bystricky, F. Lehar, and I. N. Silin, Nuovo Cimento A 1, 601 (1971).

[27] G. I. Gakh and E. Tomasi-Gustafsson, Nucl. Phys. A761, 120 (2005).

[28] E. Tomasi-Gustafsson, E. A. Kuraev, S. Bakmaev, and S. Pacetti, Phys. Lett. B 659, 197 (2008).

[29] M. Ablikim et al. (BESIII Collaboration), Chin. Phys. C 41, 063001 (2017).

[30] M. Ablikim et al. (BESIII Collaboration), Phys. Rev. D 99, 092002 (2019).

[31] J. Haidenbauer, T. Hippchen, K. Holinde, B. Holzenkamp, V. Mull, and J. Speth, Phys. Rev. C 45, 931 (1992). 